\title{
CONTINUA WHICH ARE THE SUM OF A FINITE NUMBER OF INDECOMPOSABLE CONTINUA
}

\author{
C. E. BURGESS
}

Swingle [7 ${ }^{1}$ has given the following definitions. (1) A continuum $M$ is said to be the finished sum of the continua of a collection $G$ if $G^{*}=M$ and no continuum of $G$ is a subset of the sum of the others. ${ }^{2}$ (2) If $n$ is a positive integer, the continuum $M$ is said to be indecomposable under index $n$ if $M$ is the finished sum of $n$ continua and is not the finished sum of $n+1$ continua.

Swingle has shown [7, Theorem 2] that if $n$ is a positive integer and the continuum $M$ is indecomposable under index $n$, then $M$ is the finished sum of $n$ indecomposable continua. The author has shown [2, Theorem 1] that if $n=2$ and the continuum $M$ is indecomposable under index $n$, and $G$ is a collection of $n$ indecomposable continua whose finished sum is $M$, then $G$ is the only such collection. In the present paper, it is shown that for a compact continuum, this theorem holds for any positive integer $n$. Also, there is given a necessary and sufficient condition that a compact continuum be indecomposable under index $n$.

An indecomposable continuum can be described as a nondegenerate continuum which is indecomposable under index 1 . If $n=1$, then in order that a continuum $M$ be indecomposable under index $n$, it is necessary and sufficient that $M$ contain $n+2$ points such that $M$ is irreducible about any $n+1$ of them. ${ }^{3}$ Swingle [7] has shown that it is impossible, in a certain manner, to generalize this theorem. Theorem 3 of the present paper might be considered a generalization of the necessary condition of the above theorem. However, it is easily seen that the converse of Theorem 3 is not true.

Theorems 1-5 are proved on the basis of R. L. Moore's Axioms 0 and $1_{8}$. Hence these theorems hold in any metric space. ${ }^{4}$

THEOREM 1. If $n>1$ and the compact continuum $M$ is the sum of $n$ indecomposable continua $M_{1}, M_{2}, \cdots, M_{n}$ such that, for each $i(i \leqq n)$, a composant $K_{i}$ of $M_{i}$ does not intersect $M_{1}+M_{2}+\cdots+M_{i-1}$

Presented to the Society, September 2, 1952; received by the editors May 24, 1952.

${ }^{1}$ Numbers in brackets refer to the bibliography at the end of this paper.

2 The sum of the continua of $G$ is denoted by $G^{*}$.

2 For a proof of this theorem, see [4, Theorem IV].

4 Moore's axioms are stated in [5]. The first three parts of Axiom 1 are denoted by Axiom $1_{3}$.

- If $P$ is a point of a continuum $M$, the set of all points $X$ such that $P+X$ lies in a proper subcontinuum of $M$ is called a composant of $M$. 
$+M_{i+1}+\cdots+M_{n}$, then $M$ is indecomposable under index $n$.

Proof. Suppose that there is a collection $G$ consisting of $n+1$ continua whose finished sum is $M$. No continuum of $G$ is a proper subset of one of the indecomposable continua $M_{1}, M_{2}, \cdots, M_{n}$. Hence, for each $i(i \leqq n)$, if $K_{i}$ intersects a continuum $X$ of $G$, then $X$ contains $M_{i}$. Consequently, there exist $n$ continua of $G$ such that their sum is $M$. This is contrary to the supposition that $M$ is the finished sum of the continua of $G$. Since $M$ is the finished sum of the continua $M_{1}, M_{2}, \cdots, M_{n}$, then it is indecomposable under index $n$.

Theorem 2. If $n$ is a positive integer and the compact continuum $M$ is indecomposable under index $n$, then there is only one collection of indecomposable continua whose finished sum is $M$.

Proof. By [7, Theorem 2], there is a collection $G$ consisting of $n$ indecomposable continua $M_{1}, M_{2}, \cdots, M_{n}$ such that $M$ is their finished sum. By $[3$, Theorem 1], for each $i(i \leqq n)$, some composant $K_{i}$ of $M_{i}$ does not intersect $\left(G-M_{i}\right)^{*}$. Suppose that there is a collection $G^{\prime}$ of indecomposable continua such that $G^{\prime} \neq G$ and $M$ is the finished sum of the continua of $G^{\prime}$. Let $i$ be a positive integer not greater than $n$. Some continuum $X_{i}$ of $G^{\prime}$ intersects $K_{i}$. Neither of the indecomposable continua $X_{i}$ and $M_{i}$ is a proper subset of the other. Since no proper subcontinuum of $M_{i}$ intersects both $K_{i}$ and $\left(G-M_{i}\right)^{*}$, then $X_{i}=M_{i}$. Hence $G^{\prime}=G$.

TheOREM 3. If $n>1$ and the compact continuum $M$ is indecomposable under index $n$, then there is a subset $H$ of $M$ consisting of $2 n$ points such that $M$ is irreducible about every subset of $H$ consisting of $2 n-1$ points.

Proof. Let $M_{1}, M_{2}, \cdots, M_{n}$ be $n$ indecomposable continua whose finished sum is $M$. For each $i(i \leqq n)$, let $K_{i}$ be a composant of $M_{i}$ as described in the proof of Theorem 2. There exists a subset $H$ of $M$ such that for each $i(i \leqq n), H \cdot M_{i}$ consists of two points of $K_{i}$. The set $H$ satisfies the requirements of the conclusion of Theorem 3 .

Theorem 4. If $n>1, M$ is a compact continuum, $G$ is a collection consisting of $n$ indecomposable continua whose finished sum is $M$, and $H$ is a finite set of points about which $M$ is irreducible, then $M$ is indecomposable under index $n$.

Lемма 4.1. If the hypothesis of Theorem 4 is satisfied, $X$ is a continuum of $G$, and $T$ is a component of $(G-X)^{*}$, then some composant of $X$ does not intersect $T$. 
Proof of Lemma 4.1. Suppose that every composant of $X$ intersects $T$. Then there exists a finite collection $W$ of proper subcontinua of $X$ such that $W^{*}+(G-X)^{*}$ is connected. There exists a finite collection $Y$ of proper subcontinua of $X$ such that (1) every continuum of $Y$ intersects $(G-X)^{*}$ and (2) if $X$ intersects $H$, then $Y^{*}$ contains $X \cdot H$. Since $X$ is indecomposable and $M$ is the finished sum of the continua of $G$, then $Y^{*}+W^{*}$ does not contain ${ }^{6} M-(G-X)^{*}$. Therefore, $W^{*}+Y^{*}+(G-X)^{*}$ is a proper subcontinuum of $M$ containing $H$. This is a contradiction since $M$ is irreducible about $H$.

Proof of Theorem 4. An inductive argument will be used. Suppose that Theorem 4 is not true. Let $k$ be the smallest positive integer $n$ such that if $M$ is a compact continuum satisfying the hypothesis of Theorem 4, then $M$ is not indecomposable under index $n$. By Theorem 1 , there is a continuum $X$ of $G$ such that every composant of $X$ intersects $(G-X) *$. By Lemma $4.1,(G-X)$ * is not connected. Therefore, $k>2$. The set $(G-X)^{*}$ is the sum of a finite number of mutually exclusive continua. Let $T$ be one of these continua. Since $M$ is irreducible about $H$, then $T-T \cdot X$ contains a point of $H$. By Lemma 4.1 , there is a composant of $X$ which does not intersect $T$. Let $P$ be a point of such a composant. The continuum $T+X$ is irreducible about the finite set $H \cdot T+P$. There is a positive integer $j$ less than $k$ such that $T+X$ is the finished sum of $j$ continua of $G$. Then $T+X$ is indecomposable under index $j$. By [3, Theorem 1], every continuum of $G$ which is a subset of $T+X$ contains a composant which does not intersect any other continuum of $G$ which is a subset of $T+X$. Therefore, every continuum of $G-X$ contains a composant which does not.intersect any other continuum of $G$. Let $L$ be a collection consisting of $k-1$ points such that if $Z$ is a continuum of $G-X$, then a point of $L$ belongs to a composant of $Z$ lying in $M$ $-(G-Z)^{*}$. Since, by supposition, $M$ is not indecomposable under index $k$, then there is a collection $G^{\prime}$ consisting of $k+1$ continua whose finished sum is $M$. Since the set $L$ is contained in the sum of $k-1$ continua of $G^{\prime}$, then $(G-X)^{*}$ is contained in the sum of $k-1$ continua of $G^{\prime}$. Hence there exist two continua $X_{1}$ and $X_{2}$ of $G^{\prime}$ such that each of them contains a point of $M-(G-X)^{*}$ which does not belong to any other continuum of $G^{\prime}$. Let $R$ be a domain intersecting $X_{1}$ and not intersecting $\left(G^{\prime}-X_{1}\right)^{*}+(G-X)^{*}$. Every composant of $X$ intersects $R$. Therefore, there exists a finite collection $W$ of proper sub-

- This follows from the fact that every proper subcontinuum of an indecomposable continuum $M$ is a continuum of condensation of $M[4$, Theorem II $]$ and the fact that no indecomposable continuum is the sum of a finite number of its proper subcontinua $[4$, Theorem III ]. 
continua of $X$ such that $X_{1}+W^{*}+(G-X)^{*}$ is a continuum. Let $Y$ be a finite collection of continua as described in the proof of Lemma 4.1. Since $X_{1}+Y^{*}+W^{*}+(G-X)^{*}$ is a subcontinuum of $M$ containing $H$, then $X_{1}+Y^{*}+W^{*}+(G-X)^{*}=M$. Since $X$ is indecomposable and $X_{1}+(G-X)^{*}$ contains $X-\left(Y^{*}+W^{*}\right)$, then $X_{1}+(G-X)^{*}$ contains $X$. This is impossible since $X_{1}+(G-X)^{*}$ does not contain $X_{2}$. Thus the supposition that Theorem 4 is not true has led to a contradiction.

TheOREM 5. If $n>1$, then in order that the compact continuum $M$ should be indecomposable under index $n$, it is necessary and sufficient that $M$ should be the finished sum of $n$ indecomposable continua and be irreducible about some $n$ points. ${ }^{7}$

The necessity follows from [7, Theorem 2] and [3, Theorem 2]. The sufficiency follows from Theorem 4.

THEOREM 6. If the compact continuum $M$ in the plane is the finished sum of two indecomposable continua $H$ and $K$ such that some composant of $H$ does not intersect $K$, then $M$ is indecomposable under index two.

Lемма 6.1. If the hypothesis of Theorem 6 is satisfied and $K_{1}$ and $K_{2}$ are mutually exclusive simple discs ${ }^{8}$ intersecting $K$ but not $H$, then there do not exist four mutually exclusive continua $W_{1}, W_{2}, W_{3}$, and $W_{4}$ such that, for each $i$ ( $i \leqq 4), W_{i}$ belongs to $K$, intersects $H$, and is $i r$ reducible from $K_{1}$ to $K_{2}$.

Proof of Lemma 6.1. Suppose that there do exist four such continua. Let $D$ denote the complementary domain of $K_{1}+K_{2}$. Consider the case in which $W_{3}+W_{4}$ separates $W_{1}$ from $W_{2}$ in $\bar{D}$. Let $R_{1}$ and $R_{2}$ be connected domains intersecting $H \cdot W_{1}$ and $H \cdot W_{2}$ respectively and not intersecting $K_{1}+K_{2}+W_{3}+W_{4}$. There is a composant $L$ of $H$ which intersects both $R_{1}$ and $R_{2}$ and lies in $M-K$. Then $L$ intersects $K_{1}+K_{2}+W_{3}+W_{4}$. This is a contradiction since $M-K$ does not intersect $K_{1}+K_{2}+W_{3}+W_{4}$.

Proof of Theorem 6. Suppose, on the contrary, that $M$ is the finished sum of three continua $M_{1}, M_{2}$, and $M_{3}$. One of these three continua intersects a composant of $H$ lying in $M-K$. Suppose that $M_{1}$ is such a continuum. Then it contains $H$ and intersects each of

\footnotetext{
${ }^{7}$ For an example showing that this theorem does not hold true without the condition that $M$ be irreducible about some $n$ points, see [1, p. 540]. Also, see [2, Example 1]. Sorgenfrey [6] has proved a theorem giving a necessary and sufficient condition that a compact continuum be irreducible about some $n$ points.

${ }^{8}$ In the plane, a simple closed curve together with its interior is called a simple disc.
} 
the continua $M_{2}$ and $M_{3}$. Each of the continua $M_{3}$ and $M_{1}+M_{2}$ contains a point of $K$ not belonging to the other of these two continua. Since the closure of $M-\left(M_{1}+M_{2}\right)$ is a proper subset of the indecomposable continuum $K$, then $M-\left(M_{1}+M_{2}\right)$ is not connected. Let $T_{1}$ and $T_{2}$ be two mutually separated sets whose sum is $M$ $-\left(M_{1}+M_{2}\right)$. Let $K_{1}$ and $K_{2}$ be two mutually exclusive simple discs whose interiors intersect $T_{1}$ and $T_{2}$ respectively such that $K_{1}$ and $K_{2}$ do not intersect $T_{2}+M_{1}+M_{2}$ and $T_{1}+M_{1}+M_{2}$ respectively. Since every composant of $K$ intersects both $K_{1}$ and $K_{2}$, there exist six distinct composants of $K$ each of which contains a continuum irreducible from $K_{1}$ to $K_{2}$. By Lemma 6.1, at most three of these intersect $H$, and hence three do not. Denote three which do not by $W_{1}$, $W_{2}$, and $W_{3}$. Let $D$ denote the complementary domain of $K_{1}+K_{2}$. There exist two of the continua $W_{1}, W_{2}$, and $W_{3}$ such that their sum separates the other one from $H$ in $\bar{D}$. Consider the case in which $W_{1}+W_{3}$ separates $W_{2}$ from $H$ in $\bar{D}$. Let $I$ denote the complementary domain of $K_{1}+K_{2}+W_{1}+W_{3}$ which contains the connected set $W_{2}-W_{2} \cdot\left(K_{1}+K_{2}\right)$. Since one of the sets $K_{1} \cdot W_{2}$ and $K_{2} \cdot W_{2}$ belongs to $T_{1}$ and the other to $T_{2}$, then $I \cdot W_{2}$ contains a point of the continuum $M_{1}+M_{2}$. Since $H$ is a subset of $M_{1}+M_{2}$ and does not intersect $\bar{I}$, then there is a continuum $Z$ belonging to $\bar{I} \cdot\left(M_{1}+M_{2}\right)$ and intersecting both $W_{2}$ and $W_{1}+W_{3}$. But this is impossible since $Z$ is a proper subcontinuum of $K$ intersecting two composants of $K$. Thus the supposition that $M$ is the finished sum of three continua has led to a contradiction.

THEOREM 7. If the hypothesis of Theorem 6 is satisfied, then uncountably many composants of $K$ lie in $M-H$.

This theorem follows from Theorem 6 and [3, Theorem 1].

REMARK. Neither Theorem 6 nor Theorem 7 holds true in Euclidean three-dimensional space. Let $H^{\prime}$ be the point set obtained by translating the point set $H$ of [2, Example 1] one-half unit to the left. Let $H^{\prime \prime}$ be a point set obtained by revolving $H^{\prime}$ through 90 degrees about the vertical line whose equation is $x=1 / 2$. Only one composant of $H^{\prime \prime}$ intersects $H$, but every composant of $H$ intersects $H^{\prime \prime}$. It follows from [3, Theorem 1] and Theorem 2 that the continuum $H+H^{\prime \prime}$ is not indecomposable under index two.

Added in proof. I have recently observed that Theorem 6 follows from Theorem 1 and a lemma proved by N. E. Rutt [Some theorems on triodic continua, Amer. J. Math. vol. 56 (1934) pp. 122-132 Lemma I]. I regret that I was not aware of Rutt's lemma at the time I prepared this paper. 


\section{BIBLIOGRAPHY}

1. Paul Alexandroff, Über kombinatorische Eigenschaften allgemeiner Kurven, Math. Ann. vol. 96 (1927) pp. 512-554.

2. C. E. Burgess, Continua and their complementary domains in the plane, Duke Math. J. vol. 18 (1951) pp. 901-917.

3. - Continua and their complementary domains in the plane. II, Duke Math. J. vol. 19 (1952) pp. 223-230.

4. S. Janiszewski and C. Kuratowski, Sur les continus indécomposables, Fund. Math. vol. 1 (1920) pp. 210-222.

5. R. L. Moore, Foundations of point set theory, Amer. Math. Soc. Colloquium Publications, vol. 13, 1932.

6. R. H. Sorgenfrey, Concerning continua irreducible about n points, Amer. J. Math. vol. 68 (1946) pp. 667-671.

7. P. M. Swingle, Generalized indecomposable continua, Amer. J. Math. vol. 52 (1930) pp. 647-658.

THE UNIVERSITY OF UTAH 